Ben Scholl, Xiang Gao and Michael Wehr

J Neurophysiol 99:1616-1627, 2008. First published Jan 23, 2008; doi:10.1152/jn.01172.2007

You might find this additional information useful...

This article cites 58 articles, 34 of which you can access free at: http://jn.physiology.org/cgi/content/full/99/4/1616\#BIBL

Updated information and services including high-resolution figures, can be found at: http://jn.physiology.org/cgi/content/full/99/4/1616

Additional material and information about Journal of Neurophysiology can be found at: http://www.the-aps.org/publications/jn

This information is current as of July 21, 2008 . 


\title{
Level Dependence of Contextual Modulation in Auditory Cortex
}

\author{
Ben Scholl, Xiang Gao, and Michael Wehr \\ Institute of Neuroscience, University of Oregon, Eugene, Oregon
}

Submitted 22 October 2007; accepted in final form 23 January 2008

Scholl B, Gao X, Wehr M. Level dependence of contextual modulation in auditory cortex. J Neurophysiol 99: 1616-1627, 2008. First published January 23, 2008;doi:10.1152/jn.01172.2007. Responses of cortical neurons to sensory stimuli within their receptive fields can be profoundly altered by the stimulus context. In visual and somatosensory cortex, contextual interactions have been shown to change sign from facilitation to suppression depending on stimulus strength. Contextual modulation of high-contrast stimuli tends to be suppressive, but for low-contrast stimuli tends to be facilitative. This trade-off may optimize contextual integration by cortical cells and has been suggested to be a general feature of cortical processing, but it remains unknown whether a similar phenomenon occurs in auditory cortex. Here we used whole cell and single-unit recordings to investigate how contextual interactions in auditory cortical neurons depend on the relative intensity of masker and probe stimuli in a two-tone stimulus paradigm. We tested the hypothesis that relatively low-level probes should show facilitation, whereas relatively high-level probes should show suppression. We found that contextual interactions were primarily suppressive across all probe levels, and that relatively low-level probes were subject to stronger suppression than high-level probes. These results were virtually identical for spiking and subthreshold responses. This suggests that, unlike visual cortical neurons, auditory cortical neurons show maximal suppression rather than facilitation for relatively weak stimuli.

\section{N T R O D U C T I O N}

Contextual interactions are a general feature of cortical sensory information processing. Stimuli presented outside the classical receptive field can modulate the response to stimuli at the center of the receptive field in visual, somatosensory, and auditory cortical neurons (Allman et al. 1985; Brosch and Schreiner 1997; Calford and Semple 1995; Nelson and Frost 1978; Phillips and Cynader 1985; Reale and Brugge 2000; Simons 1985; Zhang et al. 2005). These contextual interactions include both suppression and facilitation and may be involved in perceptual grouping effects such as figure-ground segmentation, contour completion, forward masking, and other aspects of visual or auditory scene analysis (Albright and Stoner 2002; Micheyl et al. 2007). In studies of visual cortex, center and surround stimuli are usually (but not always) presented simultaneously, and cortical contributions can be isolated with the use of oriented stimuli. In studies of auditory cortex, central contributions are typically isolated with the use of a sequential two-tone (forward masking) paradigm in which the first tone (termed the masker) modulates the response to the second tone (termed the probe). Sequential presentation of surround and central stimuli can also produce facilitation and suppression in visual cortex and somatosensory cortex (Lorenceau et al. 2001; Simons 1985) as well as enhanced or reduced sensitivity in

Address for reprint requests and other correspondence: M. Wehr, Institute of Neuroscience, University of Oregon, Eugene, OR 97403 (E-mail: wehr@uoregon. edu). visual and auditory psychophysical studies (Oberfeld 2007; Polat and Sagi 2006; Tadin et al. 2006; Watson and Nachmias 1977). This raises the possibility that contextual interactions, whether simultaneous or sequential, may be a general cortical phenomenon that shares important features across sensory modalities.

In primary visual cortex and primary somatosensory cortex, context-dependent enhancement and suppression appear to depend on the relative level (or contrast) of the probe (Levitt and Lund 1997; Moore et al. 1999; Polat et al. 1998; Sengpiel et al. 1997; Toth et al. 1996). For high-level or high-contrast probes, contextual stimuli tend to cause suppression. For lowlevel or low-contrast probes, contextual stimuli cause facilitation. This cross-over between facilitative and suppressive contextual interactions may optimize the tradeoff between sensitivity and specificity depending on the reliability of sensory information and has been suggested to be a general feature of cortical dynamics (Moore et al. 1999). In auditory psychophysics, similarly, forward maskers decrease the perceived loudness of the probe when the probe is higher in level than the masker, but increase the perceived loudness of the probe when the probe is lower in level than the masker (Galambos et al. 1972; Oberfeld 2007). This raises the question of whether auditory cortical neurons, like visual and somatosensory cortical neurons, exhibit a similar cross-over between facilitation and suppression depending on the relative level of the probe. In primary auditory cortex, however, contextual interactions have generally been studied at only a single probe level (Brosch and Schreiner 2000, 1997; Calford and Semple 1995; Malone and Semple 2001; Shamma and Symmes 1985; Sutter et al. 1999). It is therefore unknown whether the relative intensity of masker and probe stimuli regulates whether contextual interactions in auditory cortical neurons are facilitative or suppressive.

Several lines of evidence suggest that contextual interactions may be mediated by the subthreshold receptive field-the region over which stimuli can evoke subthreshold postsynaptic potentials - which extends beyond the classical receptive field defined by spiking responses (Bringuier et al. 1999; Kaur et al. 2004). Imaging and intracellular recordings in visual and somatosensory cortex have shown that subthreshold inputs, including both excitatory and inhibitory inputs, can be evoked from outside the classical receptive field and may be mediated by intracortical long range horizontal connections in combination with local inhibitory circuits (Bringuier et al. 1999; Hirsch and Gilbert 1991; Horikawa et al. 1997; Kaur et al. 2004; Toth et al. 1996). The cross-over between facilitation and suppression, depending on probe level, has therefore been suggested to reflect a shift in the balance of excitation and inhibition in

The costs of publication of this article were defrayed in part by the payment of page charges. The article must therefore be hereby marked "advertisement" in accordance with 18 U.S.C. Section 1734 solely to indicate this fact. 
cortical networks (Polat et al. 1998). At low probe levels, surround stimuli may primarily evoke depolarization via excitatory horizontal connections, without being able to activate local inhibition. High-level probes may recruit local inhibition, which can then be further driven by horizontal connections and may have greater influence than the excitation from those same connections (Somers et al. 1998). Alternatively, if the balance of excitation and inhibition is different between feedforward and feedback circuits (Shao and Burkhalter 1996), a shift from facilitation to suppression could arise from a shift from the dominance of feedback inputs to feedforward inputs. In the auditory system, suppressive effects from outside the classical receptive field are seen from the cochlear nucleus to the auditory cortex. The suppression seen in auditory cortex could therefore be inherited from lower levels or could involve further synaptic processing in the cortex. However, contextual interactions at the level of the subthreshold receptive field have not been well characterized in auditory cortex, and it remains unknown how excitatory and inhibitory subthreshold inputs contribute to facilitation and suppression of spiking responses (Ojima and Murakami 2002; Tai and Zador 2001; Wehr and Zador 2005).

Here we used whole cell and single-unit (extracellular) recordings to investigate how contextual interactions in auditory cortical neurons depend on the relative intensity of masker and probe stimuli in a two-tone stimulus paradigm. We hypothesized that in auditory cortex, the dependence of contextual interactions on probe level would be similar to that observed in visual and somatosensory cortex. Specifically we tested the prediction that relatively low-level probes should show facilitation, whereas relatively high-level probes should show suppression. We found that contextual interactions were primarily suppressive across all probe levels and that relatively low-level probes were subject to stronger suppression than high-level probes. These results were virtually identical for spiking and subthreshold responses. This suggests that, unlike visual or somatosensory cortical neurons, auditory cortical neurons show maximal suppression rather than facilitation for relatively weak stimuli.

\section{METHODS}

\section{Physiology}

We recorded from the left primary auditory cortex of 29 anesthetized $(30 \mathrm{mg} / \mathrm{kg}$ ketamine, $0.24 \mathrm{mg} / \mathrm{kg}$ medetomidine) rats aged 19-40 days postnatal (mean age: 26 days). All procedures were in strict accordance with the National Institutes of Health guidelines as approved by the University of Oregon Animal Care and Use Committee. Recordings were made from primary auditory cortex (A1) as determined by the frequency-amplitude tuning properties of cells and local field potentials. We recorded from all subpial depths (mean: 397 $\mu \mathrm{m}$, range: $140-800 \mu \mathrm{m}$, as determined from micromanipulator travel). For single-unit recordings, we used the cell-attached patch method (DeWeese et al. 2003), which provides excellent isolation. For whole cell recordings, we used standard blind patch-clamp methods (Wehr and Zador 2003). Internal solution contained, in mM, 140 K-gluconate, $10 \mathrm{HEPES}, 2 \mathrm{MgCl}_{2}, 0.05 \mathrm{CaCl}_{2}, 4 \mathrm{MgATP}, 0.4$ NaGTP, $10 \mathrm{Na}_{2}$ Phosphocreatine, and 10 BAPTA, pH 7.25, diluted to 290 mosM. Cell-attached and whole cell recordings were made in current clamp $(I=0)$ mode using an Axopatch 200b amplifier.

\section{Stimuli}

We presented 25-ms pure tones with 2-ms 10-90\% cosine-squared ramps, sampling rate of $200 \mathrm{kHz}$, using a 24-bit Lynx22 soundcard, a Stax SRM-717 driver and SR-303 speaker, in free-field configuration (speaker located $15 \mathrm{~cm}$ lateral to, and facing, the contralateral ear) in a sound-isolation chamber with anechoic surface treatment. This system was calibrated using a Bruel and Kjaer 2670 microphone positioned where the ear would be without the animal present. We initially characterized characteristic frequency and threshold for each cell, typically using a pseudorandomly interleaved array of tones with 16 frequencies logarithmically spaced from 1 to $40 \mathrm{kHz}$, and seven levels from 0 to $80 \mathrm{~dB}$. We use "dB" to indicate "dB SPL" throughout. We defined characteristic frequency (CF) as the frequency at which spikes (for cell-attached recordings) or synaptic potentials (for whole cell recordings) could be reliably evoked at the lowest intensity, which we in turn defined as threshold. Cells without a clear CF (due to poor tuning or responsiveness) were not included in this report. We used two different two-tone (forward masking) stimulus designs, one that used a wide range of masker frequencies and another that used a wide range of probe levels. 1) The first two-tone stimulus used maskers of 16 fixed frequencies logarithmically spaced from 1 to $40 \mathrm{kHz}$, and 5 fixed levels from 0 to $80 \mathrm{~dB}$. The masker was followed by a probe with onset 80,125 , or $200 \mathrm{~ms}$ after masker onset. We set probe frequency to the characteristic frequency for the cell, and selected two probe levels: one low level, $\sim 10-20 \mathrm{~dB}$ above threshold, and one high level, typically $80 \mathrm{~dB}$ or (for strongly nonmonotonic cells) the highest level that evoked a robust response. All masker-probe combinations were pseudorandomly interleaved. 2) The second two-tone stimulus consisted of a single masker frequency, at $\mathrm{CF}$, and a single probe frequency, also at $\mathrm{CF}$, but had seven fixed masker and probe levels from 0 to $80 \mathrm{~dB}$. For both two-tone stimulus designs, we used a 500-ms inter-stimulus interval (between the end of each probe and the onset of the next masker), and we presented a minimum of 10 repetitions of each masker-probe combination as well as 10 repetitions of each probe tone in isolation.

\section{Analysis}

Because we used multiple recording technologies and stimulus designs, we briefly summarize here the numbers of cells recorded under different conditions. Our sample consisted of 73 cells of which 18 were whole cell recordings and 55 were single-unit recordings. Of the whole cell recordings, we used the two-probe-level stimulus in 14 cells and the seven-probe-level stimulus in 7 cells (and both in 3 cells). Of the single-unit recordings, we used the two-probe-level stimulus in 31 cells and the seven-probe-level stimulus in 46 cells (and both in 22 cells). For our analysis of spiking responses, we pooled whole cell and single-unit recordings, producing a sample of 45 cells for the two-probe-level stimulus and 53 cells for the seven-probe-level stimulus.

We extracted spike times from whole cell and single-unit recordings by high-pass filtering and thresholding. We digitally removed spikes from whole cell recordings to isolate subthreshold responses by clipping spikes at a fixed threshold of $20 \mathrm{mV}$ above the mean membrane potential (averaged across the entire recording) and then low-pass filtering at $100 \mathrm{~Hz}$. This provides a reasonable estimate of the subthreshold membrane potential response, uncontaminated by spikes (Azouz and Gray 1999; Carandini and Ferster 2000) (see Fig. 1 for an example of raw and subthreshold traces). We quantified spiking responses using the spike count in a window following tone onset (matched to response latency and duration for each cell). We quantified spontaneous firing rates using the spike count in a window of matched duration prior to tone onset. We quantified depolarizing subthreshold responses by measuring peak membrane potential in a similarly defined window (relative to the "resting" membrane potential measured during a baseline period $40-50 \mathrm{~ms}$ prior to stimulus onset and averaged across an entire stimulus protocol). We quantified 


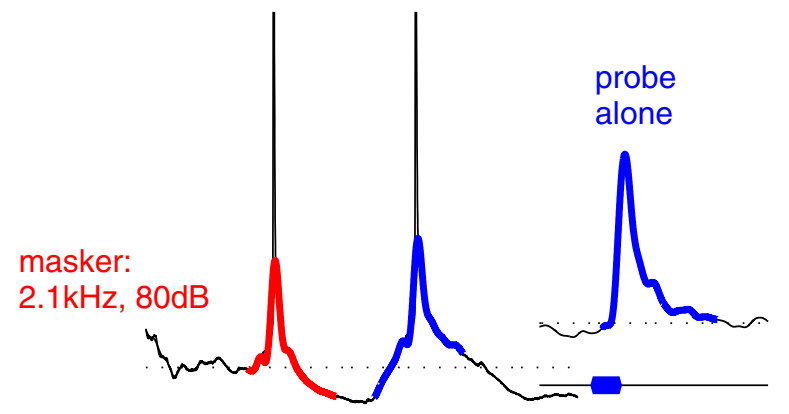

masker:

$7.2 \mathrm{kHz}, 80 \mathrm{~dB}$

probe:

$6 \mathrm{kHz}, 80 \mathrm{~dB}$
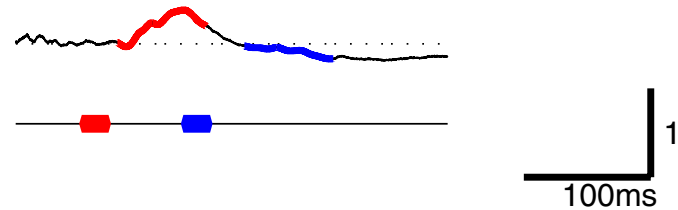

$10 \mathrm{mV}$

FIG. 1. Examples of spiking and subthreshold responses to 2-tone stimuli. The 1st tone (red) is termed the masker and the following tone (blue) is termed the probe. Spike counts for both single-unit and whole cell recordings were measured in time windows following the masker (red region) and probe (blue region). In whole cell recordings, we also isolated subthreshold responses by digitally removing spikes (red and blue traces) and measuring peak depolarization in the same time windows. Inset shows the subthreshold response (with spikes removed) to the probe in isolation, averaged across trials. Masker frequency and level were varied, whereas probe frequency was fixed at the characteristic frequency of the cell (here $6 \mathrm{kHz}$ ) and presented at different levels. Note that the $2.1-\mathrm{kHz}$ masker evoked a large response but caused no suppression; the $7.2-\mathrm{kHz}$ masker [closer to characteristic frequency (CF)] evoked a weak response but caused total suppression. Tones were $25 \mathrm{~ms}$ in duration; interval between masker onset and probe onset was $80 \mathrm{~ms}$ for this and all subsequent figures.

tone-evoked hyperpolarization by measuring minimum membrane potential for isolated tone responses, relative to rest, in a fixed window 0-350 ms after tone onset. We quantified suppressive bandwidth as the contiguous region in which spiking responses were reduced below a criterion of spontaneous spike count $+20 \%$ of peak spike count (Sutter and Schreiner 1991; Sutter et al. 1999).

We quantified contextual modulation with a measure (contextual modulation index, CMI) defined as

$$
\mathrm{CMI}=\frac{R_{M+P}-R_{P}}{R_{M+P}+R_{P}}
$$

where $R_{\mathrm{M}+\mathrm{P}}$ is the response (either spike count or peak depolarization) to the probe following a masker, and $R_{\mathrm{P}}$ is the response (either spike count or peak depolarization) to the probe presented in isolation. This measure varies from -1 (complete suppression) to +1 (maximal facilitation) and has a value of 0 when the masker has no effect on the probe. This measure is well behaved for nonnegative responses but can be ill-conditioned for negative responses. Spike counts were by definition always nonnegative, but in rare cases, spontaneous membrane potential fluctuations caused negative peak depolarizations, and we therefore truncated CMI at \pm 1 for subthreshold responses. For statistical group comparisons we used the paired $t$-test except where otherwise noted. Error bars indicated SEs.

\section{R E S U L T S}

\section{Spiking responses}

We measured contextual modulation of neurons in A1 using a two-tone stimulus paradigm. This stimulus consisted of two brief ( $25 \mathrm{~ms}$ ) pure tones separated by an 80 -ms interval; we refer to the first tone as the masker and the second tone as the probe. An example of subthreshold and spiking responses of an auditory cortical neuron to masker and probe tones is shown in Fig. 1. In this neuron, a 2-kHz masker had little effect on the response to the $6-\mathrm{kHz}$ probe, whereas a $7-\mathrm{kHz}$ masker completely suppressed the response to the same probe. Thus strong masking was evoked by tones near the characteristic frequency (6 kHz for this cell) as was typical for the cells in our sample and consistent with previous reports (Brosch and Schreiner 1997; Calford and Semple 1995; Sutter et al. 1999; Tai and Zador 2001).

This also illustrates that strong masking can be evoked even by tones that produce weak subthreshold responses and are therefore outside of the receptive field as defined by spiking responses. This phenomenon has been referred to as lateral suppression or lateral inhibition (Brosch and Schreiner 1997; Calford and Semple 1995), although as illustrated in Fig. 1, we observed that this response suppression was uncorrelated with the strength of hyperpolarizing inhibition.

To characterize the contextual modulation of spiking responses, we measured spike counts evoked by the probe stimulus (blue regions in Fig. 1) in 45 neurons (14 whole cell recordings and 31 single-unit recordings). An example is shown in Fig. 2. The spiking responses evoked by the masker components of the two-tone sequences are shown in Fig. $2 A$, revealing the $\mathrm{V}$-shaped receptive field. Figure $2 B$ shows the spiking responses to a high-level $(80 \mathrm{~dB})$ probe at the characteristic frequency $(\mathrm{CF})$ for this neuron $(3.4 \mathrm{kHz})$. The maskers evoked a V-shaped region of suppression, centered on the receptive field of the cell. We then tested whether the same maskers evoked facilitation for a relatively low-level probe by reducing the probe level to $30 \mathrm{~dB}$ (chosen to be $\sim 10 \mathrm{~dB}$ above threshold for this cell). When we used this low-level probe (Fig. 2C), the extent of the suppressive region was in fact larger for the responses to the low-level probe than for responses to the high-level probe.

This was true across all cells in our sample. To average the response profiles across cells, we aligned response profiles to the characteristic frequency for each cell. Figure $3 A$ shows the spiking responses to the maskers, averaged across the population ( $n=45$ cells). Figure $3, B$ and $C$, shows the spiking responses to high- and low-level probes, averaged across the population. The high-level probes were typically $80 \mathrm{~dB}$ or for strongly nonmonotonic cells were the highest level which evoked a response (mean: $76 \mathrm{~dB}$, range: $30-80 \mathrm{~dB}$ ). The low-level probe levels were chosen to be the lowest level which evoked a robust response, typically 10-20 dB above threshold (mean: $10 \mathrm{~dB}$, range: $-10-40 \mathrm{~dB}$ ). Suppressive regions were centered on the $\mathrm{CF}$ of each cell. Across cells, the extent of the suppressive region was smaller for the high-level probe and larger for the low-level probe. To quantify this effect, we measured the bandwidth of the suppressive zone at 20 and $40 \mathrm{~dB}$ above threshold. Suppressive bandwidth was significantly greater for low-level probes than for high-level probes (by $0.76 \pm 0.24$ octaves at $20 \mathrm{~dB}$ above threshold, $P<$ 0.001 ; by $0.76 \pm 0.21$ octaves at $40 \mathrm{~dB}$ above threshold, $P<$ 0.001).

High-level probes typically evoked a higher firing rate than low-level probes, and the raw spike counts shown in Fig. 3, $B$ and $C$, include this effect as well as the difference in the 

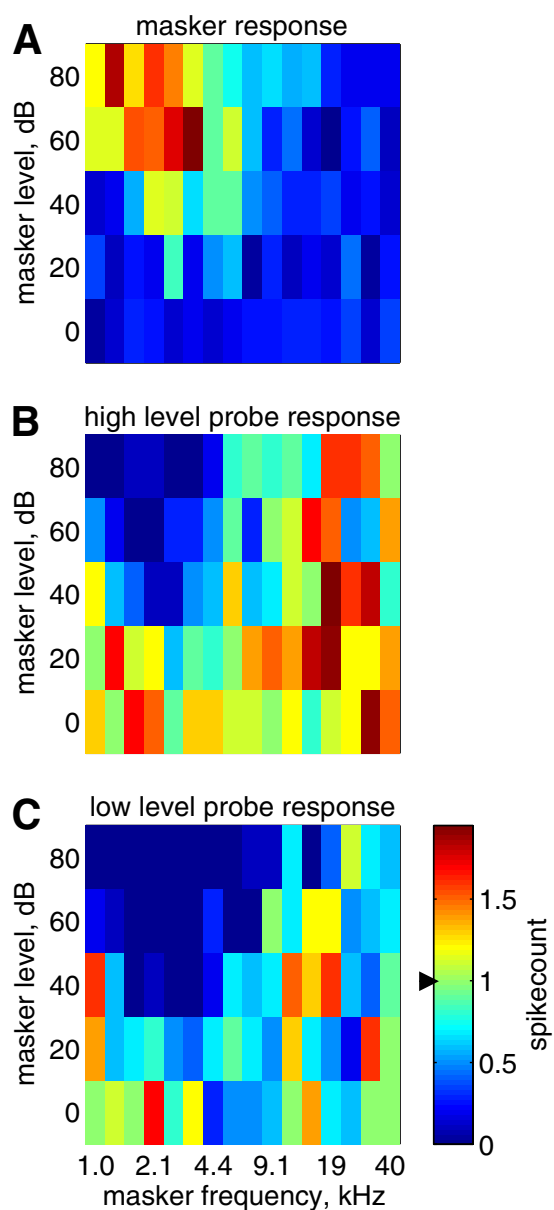

FIG. 2. Forward suppression of spiking responses was greater for low probe levels than for high probe levels: example cell. A: spiking responses evoked in an auditory cortical neuron by the masker component of the 2-tone sequence, showing the spiking receptive field. CF was $3.4 \mathrm{kHz}$ and threshold was $20 \mathrm{~dB}$. $B$ : spiking responses evoked by a high level probe $(80 \mathrm{~dB}, 3.4 \mathrm{kHz})$. Note the blue region of suppression centered on CF. In this neuron, there was also a small region of facilitation above $\mathrm{CF}$. $C$ : spiking responses evoked by a low level probe $(30 \mathrm{~dB}, 3.4 \mathrm{kHz})$. Note the greater extent of the suppressive region for the low level probe. Responses to the probes in isolation are indicated by the arrowhead on color bar (both high and low level isolated probes evoked the same spike count).

amount of contextual modulation. To isolate the contextual modulation effect, we computed a contextual modulation index (CMI, see METHODS) by comparing responses to the probe following a masker with responses to the probe in isolation. This measure varies from -1 (complete suppression) to +1 (maximal facilitation) and has a value of 0 when there is no contextual modulation. Figure $3, D$ and $E$, shows this measure of contextual modulation averaged across the population. The extent and depth of suppression were greater for low-level probes than for high-level probes. Figure $3 F$ shows the mean contextual modulation index, averaged across all tones and cells, indicating that low-level probes were significantly more suppressed than high-level probes $(P<0.001)$. To quantify the extent of the suppressive zone, we counted the fraction of tones that evoked a CMI significantly below zero. Across the population, the extent of the suppressive zone was significantly greater for low-level probes $(36 \%)$ than for high-level probes $(23 \%, P<0.05)$. Across the population, the extent of facilitation (quantified as the fraction of tones which evoked a CMI significantly greater than 0 ) was small $(1 \%)$ and was not significantly different between high- and low-level probes.

These results suggest that contextual modulation of spiking responses is largely suppressive and is greater for low-level probes than for high-level probes. This casts doubt on the hypothesis that contextual modulation is facilitative for lowlevel probe levels. We wondered, however, whether the use of only two probe levels (1 high and 1 low level, near threshold) might have led us to miss facilitation occurring for a narrow range of probe levels (Brosch and Schreiner 2000). We therefore varied probe level across a wide range to systematically explore the effect of probe level on contextual modulation. Because of the combinatorial increase in the time required to present all possible combinations of masker and probe, we used a single masker frequency (at the characteristic frequency of the cell). Figure $4 A$ shows the spiking response to the probe as a function of probe level averaged across the population ( $n=53$ cells). As masker level was increased (from blue to red lines), probe responses were progressively suppressed. For very low-level probes, this suppression was poorly defined because there was only a weak response to the probe in isolation. As probe level was increased, the amount of suppression first increased as the probe response became larger and suppression was measurable. As probe level was increased further, the amount of suppression then decreased, consistent with a competitive interaction between the probe response and the suppression induced by the masker. Figure $4 B$ shows the amount of suppression (CMI) as a function of probe level and masker level. The amount of suppression depended strongly on masker level but more weakly on probe level. This can be clearly seen in Fig. 4C, which shows the amount of suppression for each masker level averaged across probe levels. The amount of suppression depended strongly on masker level. Figure $4 D$ shows the amount of suppression for each probe level averaged across masker levels. The amount of suppression showed a U-shaped dependence on probe level with maximal suppression evoked at intermediate probe levels. This suggests that suppression was poorly defined for very-lowlevel probes, was maximal for intermediate probes, and was reduced for very-high-level probes. The dependence of suppression on both masker and probe levels was highly significant $(P<0.001,2$-way ANOVA).

We did not observe facilitation at the population level, as can be seen in Figs. 3 and 4. However, 51\% of the cells (37/73) showed statistically significant facilitation for one or more masker-probe combinations. To quantify the extent of facilitation and suppression, we counted the fraction of tones that evoked a CMI significantly greater or less than 0. Typically, the extent of facilitation was quite small (mean: $2 \%$, range: $0-22 \%, n=73$ cells). In contrast, all cells showed statistically significant suppression for one or more tones with a much greater extent (mean: $30 \%$, range: $0-94 \%, n=73$ cells). The extent of facilitation and the extent of suppression were negatively correlated across cells (regression slope $=-0.05, r^{2}=$ $0.09, P<0.001$ ), suggesting that cells that showed greater suppression tended to show less facilitation, but there did not appear to be any distinct subpopulations of cells. To assess which tones were most likely to evoke facilitation or suppression, we measured the number of cells showing statistically significant facilitation or suppression for each masker and probe combination. As shown in Fig. 5, $A$ and $B$, suppression 
A

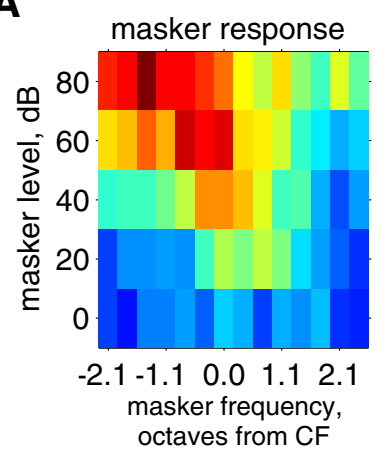

B

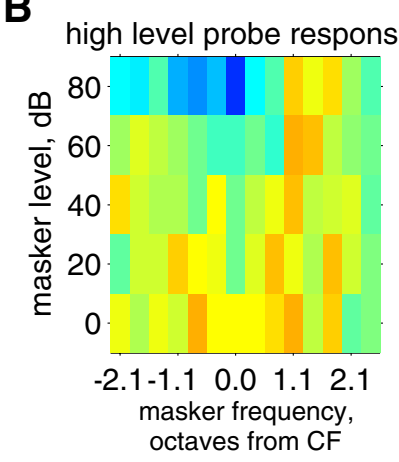

D

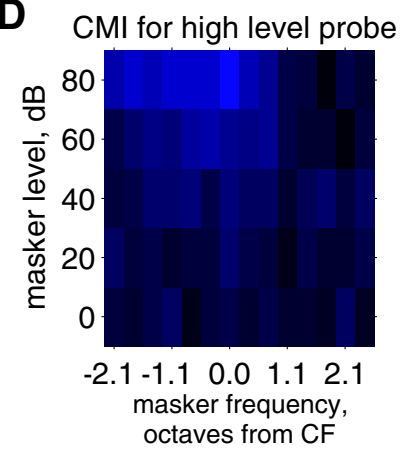

C

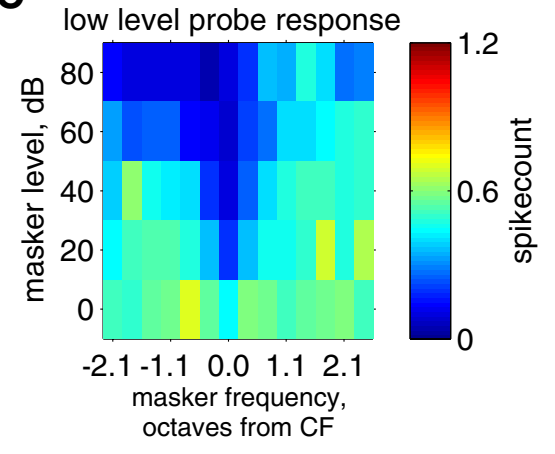

E

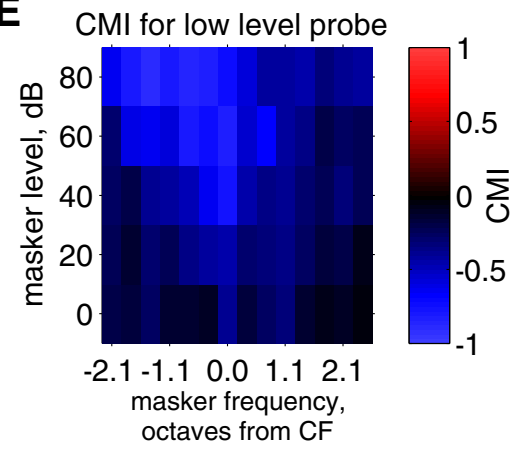

$\mathbf{F}$

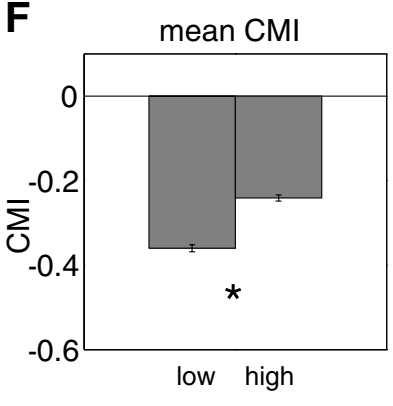

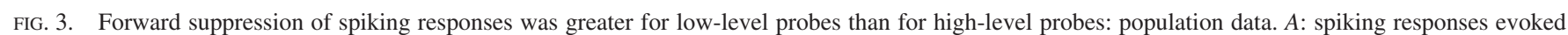

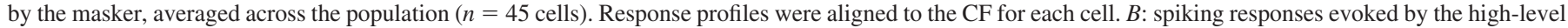

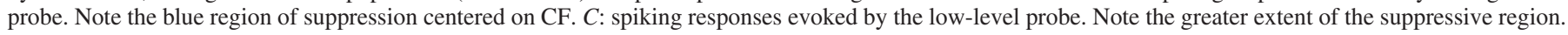

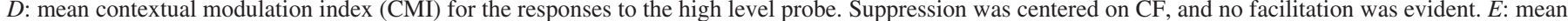

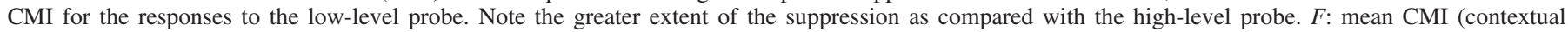
modulation index) averaged across all tones and cells, for low- and high-level probes. Suppression was stronger for low-level probes.

was centered on the receptive field and depended on both masker and probe level. The percentage of cells showing significant suppression was highest for high masker levels and for relatively low-level probe levels. In contrast, facilitation was much less prevalent and did not depend on masker frequency, masker level, or probe level (Fig. 5, $C$ and $D$ ). When we systematically varied probe levels over a wide range, we again observed that the greatest incidence of suppression was for high masker levels and for intermediate probe levels (Fig. $5 E$ ). The incidence of suppression was reduced for very-low- level and very-high-level probes. The incidence of facilitation was low (Fig. $5 F$ ) and did not depend on masker or probe levels. The incidence of facilitation was similar for monotonic cells $(27 / 56,48 \%)$ and for nonmonotonic cells $(10 / 17,59 \%)$.

Although the predominance of suppression we observed is consistent with previous work (Brosch and Schreiner 1997, 2000; Calford and Semple 1995), we wondered whether the relative strength or extent of facilitation might depend on the interval between the masker and probe. To address this issue, we varied the interval between masker and probe over the
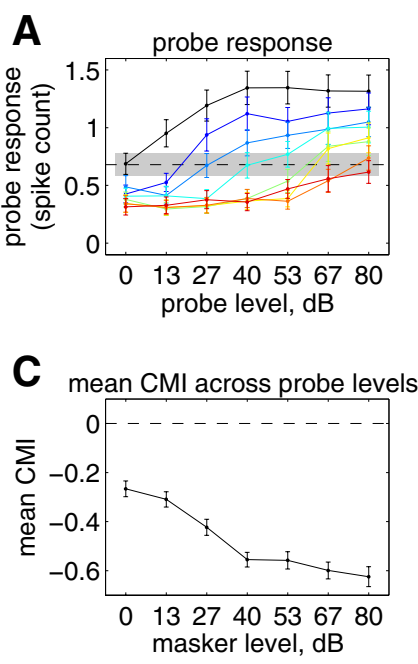
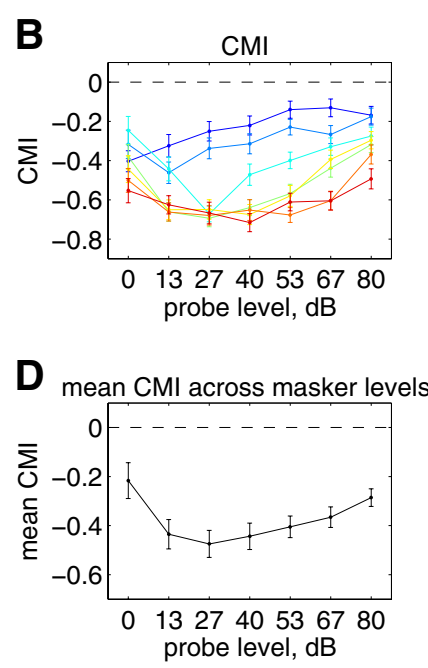

masker level

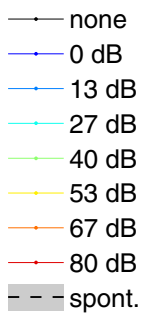

FIG. 4. Forward suppression depended on both masker and probe levels. A: spiking responses evoked by the probe as a function of probe level, for a wide range of masker levels, averaged across the population $(n=53$ cells). Different masker levels are shown by colored lines (masker levels indicated in legend at right, "none" indicates the response to the probe in isolation). $B$ : mean CMI as function of probe level, for a range of masker levels, averaged across the population. Different masker levels are indicated by colored lines. $C$ : mean $\mathrm{CMI}$ as a function of masker level, averaged across probe levels. Suppression depended strongly on masker level. $D$ : mean CMI as a function of probe level, averaged across masker levels. Suppression was strongest for intermediate probe levels. 

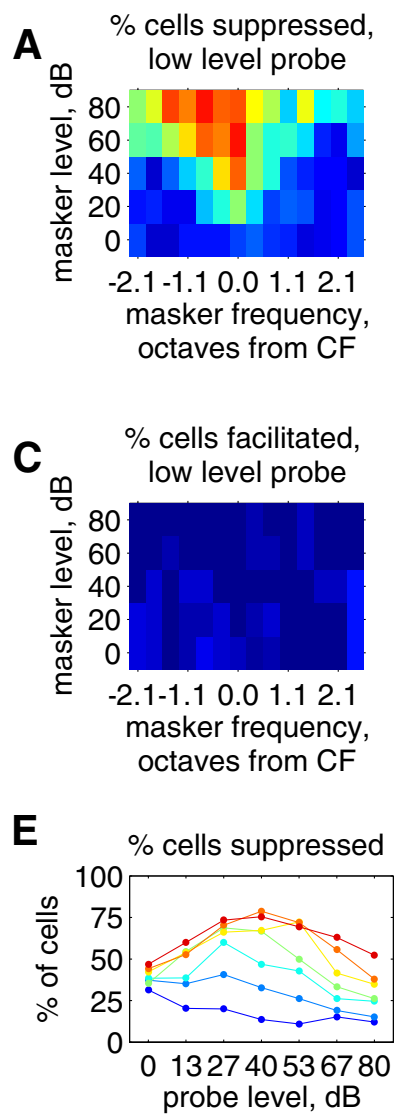
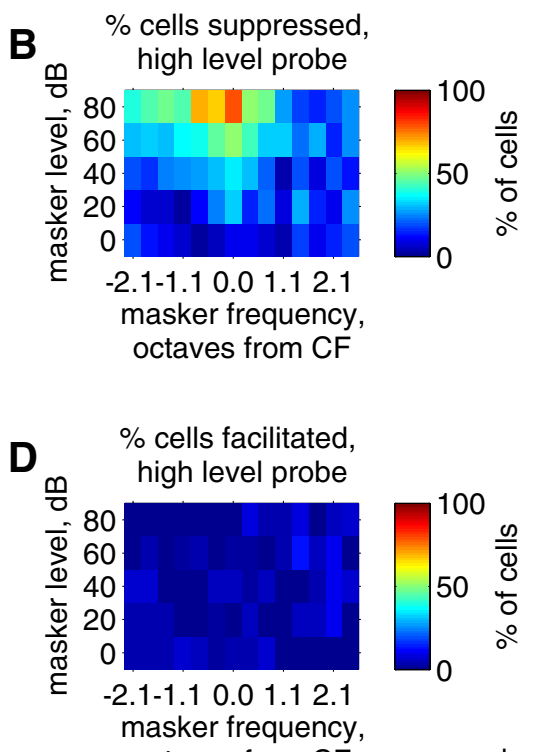

masker level

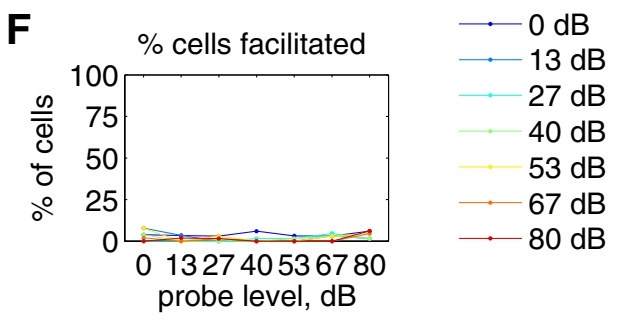

FIG. 5. Incidence of suppression was greater for low-level probes than for high-level probes. A: percentage of cells that showed significant suppression of the response to the low-level probe, as a function of masker frequency and level. $B$ : percentage of cells that showed significant suppression for the highlevel probe. $C$ : percentage of cells that showed significant facilitation for the low-level probe. $D$ : percentage of cells that showed significant facilitation for the high-level probe. Incidence of facilitation was low and did not depend on stimulus parameters. $E$ : percentage of cells that showed significant suppression, as a function of masker and probe level. Incidence of suppression was highest for high masker levels and intermediate probe levels. $F$ : percentage of cells that showed significant facilitation, as a function of masker and probe level. range $80-200 \mathrm{~ms}$ in a subset of cells. This range of intervals is within the range for which facilitation has been previously reported (Brosch and Schreiner 2000). We found that increasing the interval from $80 \mathrm{~ms}$ to either $125 \mathrm{~ms}$ ( $n=13$ cells) or $200 \mathrm{~ms}(n=10$ cells $)$ did not significantly increase the extent of facilitation. Increasing the interval to $200 \mathrm{~ms}$ (but not 125 ms) significantly decreased the extent of suppression $(P<$ 0.001 , Wilcoxon signed-rank test), consistent with previous reports that forward suppression in auditory cortical neurons lasts $\sim 150 \mathrm{~ms}$ (Brosch and Schreiner 1997). Thus the limited extent of facilitation, and the absence of any dependence on masker or probe level, was true over a range of intervals between masker and probe.

\section{Subthreshold responses}

These results suggest that the contextual modulation of spiking responses in primary auditory cortical neurons remains suppressive across a wide range of probe levels, in contrast to the facilitation seen for low-contrast central stimuli in primary visual cortex (Polat et al. 1998; Toth et al. 1996). Because contextual modulation is thought to be mediated by subthreshold inputs (Moore et al. 1999; Polat et al. 1998), we wondered whether we might see facilitation at the level of subthreshold depolarizations. Similarly, we wondered whether suppression might be mediated by hyperpolarizing inhibition or if we could observe interactions between facilitation and suppression at the level of the membrane potential. To address these questions, we recorded membrane potential responses to two-tone stimuli using whole cell methods. To analyze contextual effects separately for spikes and subthreshold inputs, we removed spikes from membrane potential traces using a spike clipping procedure (Azouz and Gray 1999; Carandini and Ferster 2000) [Fig. 1, see METHODS; spiking data from whole cell recordings are included in the preceding analysis (Figs. 3 and 4)]. We refer to the resulting nonspiking component of the membrane potential response as the subthreshold response (even though that stimulus could have evoked a spike).

An example of subthreshold responses to two-tone stimuli is shown in Fig. 6A with masker (red) and probe (blue) regions indicated. To quantify probe responses, we measured peak depolarization in a window following probe onset (blue regions). The peak depolarization in response to the masker is shown in Fig. $6 B$, illustrating the $\mathrm{V}$-shaped subthreshold receptive field for this cell. Figure $6 C$ shows the peak depolarization in response to a high-level $(80 \mathrm{~dB})$ probe at the $\mathrm{CF}$ for this neuron $(20 \mathrm{kHz})$, and $D$ shows the peak depolarization in response to a low-level $(0 \mathrm{~dB})$ probe at the same frequency. A $\mathrm{V}$-shaped region of suppression, centered on the receptive field of the cell, can be seen for the subthreshold responses to both probe levels. As with spiking responses (Figs. 2 and 3), the extent of this subthreshold suppression was smaller for the high-level probe and larger for the low-level probe.

This was true across all cells in our sample. Figure $7 A$ shows the subthreshold responses to the masker, aligned to the characteristic frequency for each cell and averaged across the population ( $n=14$ cells). Figure $7, B$ and $C$, shows the subthreshold responses to the low- and high-level probes, averaged across the population. Across cells, the suppressive region was smaller for the high-level probe and larger for the low-level probe. Figure 7, $D$ and $E$, shows the CMI computed 
A

high level probe response

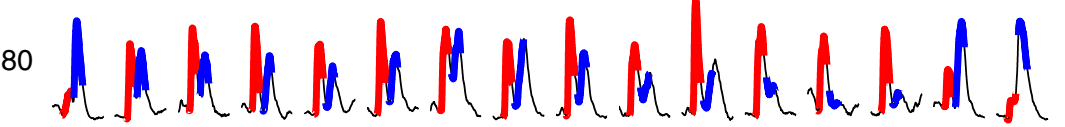

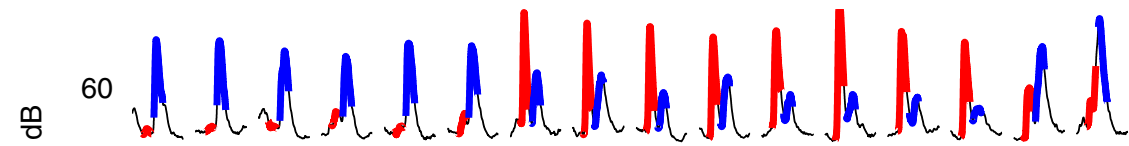

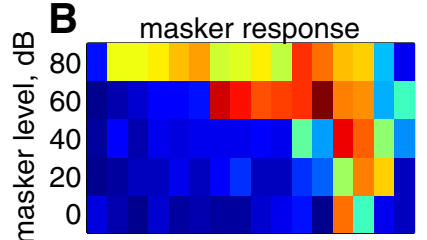

C high level probe response

高

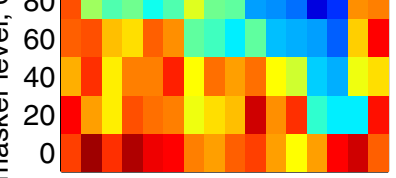

low level probe response

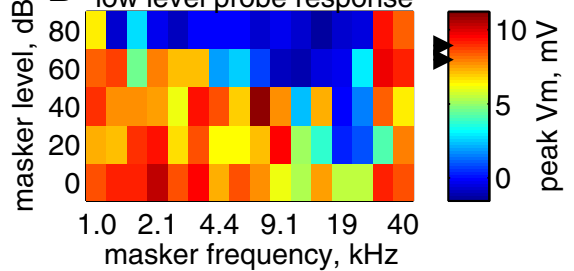

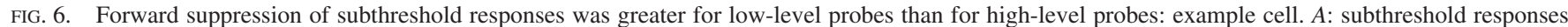

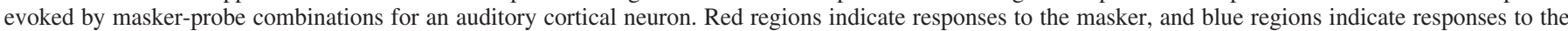

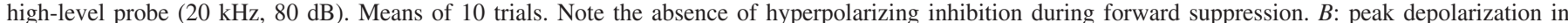

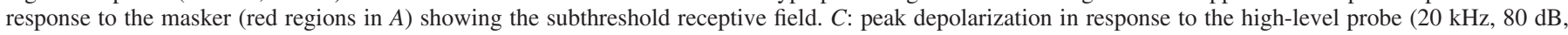

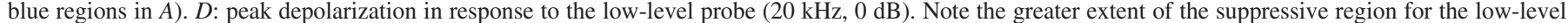
probe. Responses to the probes in isolation are indicated by arrowheads on color bar.

A

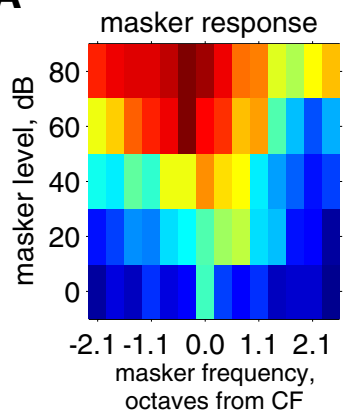

B

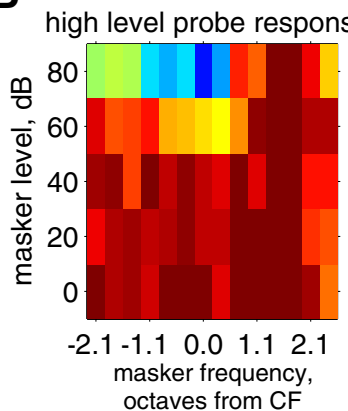

D $\mathrm{CMI}$ for high level probe

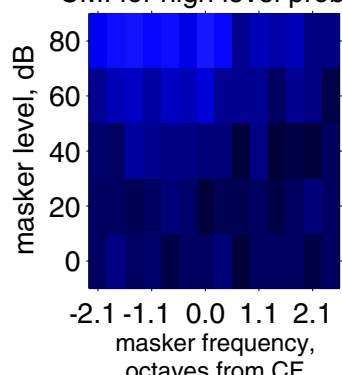

C

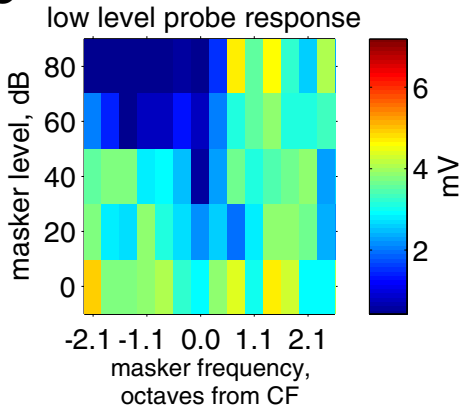

E

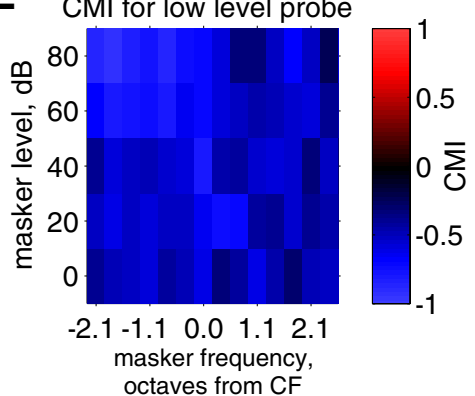

$\mathbf{F}$

$\mathbf{F} \quad$ mean $\mathrm{CMI}$

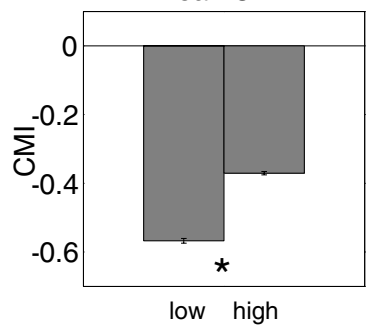

FIG. 7. Forward suppression of subthreshold responses was greater for low-level probes than for high-level probes: population data. A: subthreshold responses

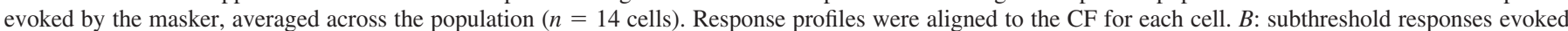

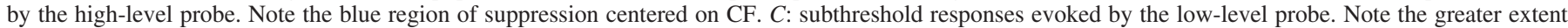

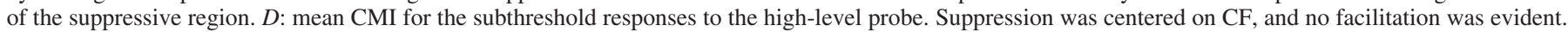

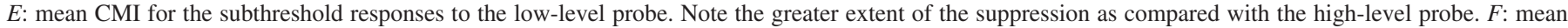
CMI for subthreshold responses, averaged across all tones and cells, for low- and high-level probes. Suppression was stronger for low-level probes. 

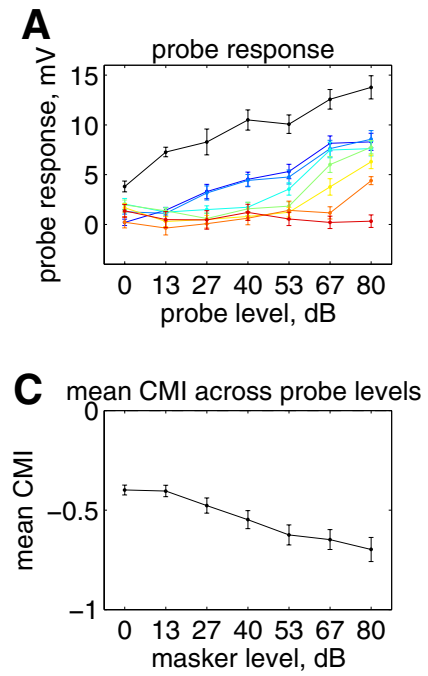
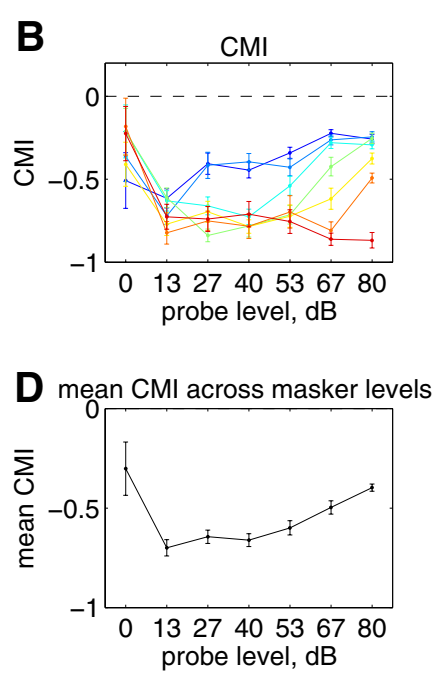

masker level

- none

$-0 \mathrm{~dB}$

$-13 \mathrm{~dB}$

$-27 \mathrm{~dB}$

$40 \mathrm{~dB}$

$53 \mathrm{~dB}$

$67 \mathrm{~dB}$

$80 \mathrm{~dB}$

FIG. 8. Forward suppression of subthreshold responses depended on both masker and probe levels. A: subthreshold responses evoked by the probe as a function of probe level, for a wide range of masker levels, averaged across the population ( $n=7$ cells). Conventions as in Fig. 4. $B$ : mean CMI as function of probe level, for a range of masker levels, averaged across the population. $C$ : mean $\mathrm{CMI}$ as a function of masker level, averaged across probe levels. Suppression depended strongly on masker level. $D$ : mean $\mathrm{CMI}$ as a function of probe level, averaged across masker levels. Suppression was strongest for intermediate probe levels. from peak depolarizations, aligned to characteristic frequency, and averaged across cells. The extent of suppression was smaller for the high-level probe and larger for the low-level probe. This was also true of the spiking responses of these cells (data not shown separately, but are included in the analysis shown in Fig. 3). Figure $7 F$ shows the mean contextual modulation index for peak depolarization, averaged across all tones and cells, indicating that low-level probes were significantly more suppressed than high-level probes. The extent of suppression was not significantly different for subthreshold responses as compared with spiking responses. Thus the properties of suppression of subthreshold responses were markedly similar to those of spiking responses (compare Figs. 3 and 7).

As before, we wondered whether the absence of facilitation for low-level probes was due to our use of only two probe levels ( 1 high level and 1 near threshold). We therefore examined contextual modulation of subthreshold responses across a wide range of probe levels by combining multiple masker and probe levels with a single masker and probe
A

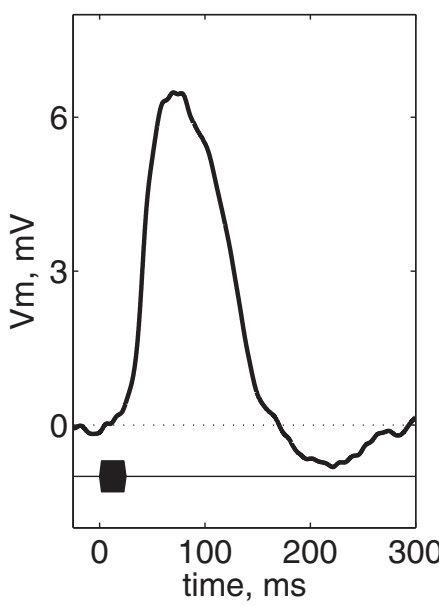

B

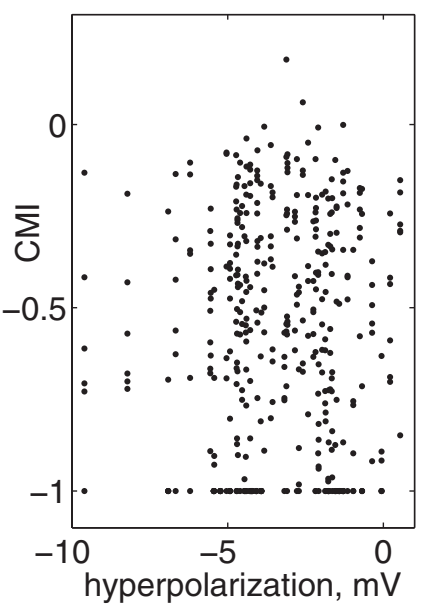

FIG. 9. Hyperpolarization and forward suppression were uncorrelated. $A$ : grand average subthreshold response evoked by a CF tone in isolation $(n=$ 15 cells). $B$ : for each cell, suppression (CMI) evoked by a CF masker is plotted against the hyperpolarization evoked by that $\mathrm{CF}$ tone in isolation. Across all cells and levels, suppression was uncorrelated with hyperpolarization $\left(r^{2}=\right.$ 0.0 , n.s.). frequency. Similar to spiking responses to these stimuli (Fig. 4), subthreshold responses to the probe were progressively suppressed as masker levels were increased (Fig. 8A). The amount of suppression, as quantified by the CMI, is shown in Fig. $8 B$ as a function of probe level and masker level. Suppression depended strongly on masker level but more weakly on probe level. Figure $8 C$ shows the amount of suppression for each masker level averaged across probe levels. The amount of suppression depended strongly on masker level. Figure $8 D$ shows the amount of suppression for each probe level, averaged across masker levels. The amount of suppression showed a U-shaped dependence on probe level, because suppression was poorly defined for very low-level probes, was reduced for very high level probes, and was maximal for intermediate probe levels. The dependence of suppression on both masker and probe levels was highly significant $(P<0.001,2$-way ANOVA).

We wondered whether this forward suppression might be mediated by cortical synaptic inhibition as has been widely proposed (Brosch and Schreiner 1997; Calford and Semple 1995; Tan et al. 2004). We observed tone-evoked hyperpolarization for one or more tones in about $2 / 3$ of the population (13/18), consistent with the presence of hyperpolarizing synaptic inhibition in a subset of auditory cortical neurons (Ojima and Murakami 2002). Figure $9 A$ shows the population average membrane potential response to $\mathrm{CF}$ tones presented in isolation. The population average tone-evoked hyperpolarization had a maximal depth of $0.9 \mathrm{mV}$ and lasted about $300 \mathrm{~ms}$ after tone onset. If hyperpolarizing inhibition were a mechanism of forward suppression, we would expect to observe a correlation between the depth of hyperpolarization and the strength of suppression. Across all tones and cells, however, the forward suppression evoked by a masker tone (as quantified by CMI) showed no correlation with the magnitude of hyperpolarization evoked by that same tone in isolation (Fig. 9B). (The hyperpolarization evoked by a masker presented before a probe could not be measured because it overlapped in time with the probe-evoked response). Hyperpolarization was also uncorrelated with peak tone-evoked depolarization. Forward suppression (as quantified by CMI) was significantly correlated with the peak depolarization evoked by the masker $(P<0.001, n=$ 18 cells), as well as negatively correlated with the distance of 
the masker from $\mathrm{CF}(P<0.01, n=14$ cells). Thus hyperpolarizing inhibition appears unlikely to mediate forward suppression, which instead appears to depend on the strength of the response to the masker and its distance from $\mathrm{CF}$.

\section{I S C U S S I O N}

In the auditory cortex, spectrotemporal interactions between two brief tones separated in time are known to depend strongly on the frequency and intensity of the first tone, but it remains unknown how these interactions depend on the intensity of the second tone. Here we have tested the hypothesis that, as in somatosensory and visual cortical neurons, the relative intensity of the masker and probe tones regulates whether spectrotemporal interactions are facilitative or suppressive. We used a two-tone, forward masking stimulus paradigm in which we systematically varied the frequency and intensity of the masker and varied the intensity of the probe. We examined the resulting suppression and facilitation of both spiking and subthreshold responses of auditory cortical neurons recorded using single-unit and whole cell methods. We found that contextual interactions were primarily suppressive and that this suppression was stronger for low and intermediate probe levels and weaker for high probe levels. The incidence of facilitation was low and did not depend on the frequency or intensity of maskers or probes. Moreover, these results were virtually identical for spiking and for subthreshold responses. Our main conclusion is therefore that the relative intensity of the masker and probe tones has no effect on whether spectrotemporal interactions are facilitative or suppressive. Rather suppression increased with increasing masker levels and decreased with increasing probe levels, consistent with a competitive interaction between the probe response and the suppression induced by the masker.

In the visual cortex, the sign of contextual modulation (i.e., facilitation or suppression) depends on the contrast of the central stimulus. For low-contrast central stimuli, surround stimuli cause facilitation, whereas for high-contrast central stimuli, surround stimuli cause suppression (Polat et al. 1998; Sengpiel et al. 1997; Toth et al. 1996). The facilitation is thought to depend on depolarizing synaptic inputs that can be evoked by stimuli outside the classical receptive field, as demonstrated by whole cell recordings (Bringuier et al. 1999) and by imaging (Toth et al. 1996). These depolarizing synaptic inputs may arise from long-range horizontal intracortical connections (Angelucci et al. 2002; Hirsch and Gilbert 1991). Similarly, in the somatosensory cortex, stimulation of nonprincipal whiskers evokes depolarizing inputs, which are thought to facilitate responses to weak stimulation of the principal whisker (Ghazanfar and Nicolelis 1997; Moore and Nelson 1998). In contrast, simultaneous stimulation of multiple whiskers causes suppression (Brumberg et al. 1996; CastroAlamancos 2002; Higley and Contreras 2005, 2007; Simons 1985). This cross-over from facilitation to suppression, depending on the contrast or intensity of the central (probe) stimulus, has been suggested to be a general property of cortical dynamics. Functionally, this cross-over has been interpreted in terms of the trade off between sensitivity and specificity (Moore et al. 1999). At low intensities, cortical circuitry may act to increase sensitivity by means of facilitation, to enhance detection. At higher intensities, cortical cir- cuitry may act to increase specificity by means of suppression, reducing receptive field size (and thereby enhancing stimulus selectivity) to improve discrimination. However, we found no evidence for such a cross-over in the sign of contextual modulation in auditory cortex. Indeed the strength and incidence of suppression were increased as probe levels were decreased with no change in the strength or incidence of facilitation. These results therefore suggest that in quiet (i.e., in the absence of contextual stimuli), auditory cortical circuitry has maximal sensitivity and is optimized for detection. In the presence of context, however, auditory cortical circuitry acts to suppress responses and thereby enhance selectivity regardless of stimulus intensity.

The picture that emerges from these results is that the strength and extent of suppression are highly correlated with the stimulus properties that most strongly drive a neuron. We found, as have others, that suppression was stronger for maskers that were higher level and closer to characteristic frequency (Bartlett and Wang 2005; Brosch and Schreiner 1997; Calford and Semple 1995; Malone and Semple 2001; Shamma and Symmes 1985). Similarly for other stimulus parameters such as binaural levels or spatial location, maskers cause stronger suppression the closer they are to a neuron's preferred stimulus (Reale and Brugge 2000; Zhang et al. 2005). We found that increasing the intensity of the probe reduced the amount of suppression, consistent with a straightforward competitive interaction between the probe response and the suppression induced by the masker. Suppression was not correlated with the amount of tone-evoked hyperpolarization. Taken together, these results are consistent with the idea that synaptic depression contributes to forward suppression (Wehr and Zador 2005) and that the degree of suppression is in part determined by the amount of overlap in the synapses activated by the masker and by the probe.

Simultaneously presented tone pairs can cause two-tone suppression in the basilar membrane and auditory nerve, but these and other peripheral contributions have generally decayed by $\sim 80 \mathrm{~ms}$ after masker onset (Harris and Dallos 1979; Patuzzi et al. 1984). We therefore used a minimum maskerprobe interval of $80 \mathrm{~ms}$ to isolate central contributions to contextual interactions, as have others (Calford and Semple 1995). This approach differs from the simultaneous contextual stimuli typically used in studies of visual cortex. There, cortical contributions can be isolated with the use of oriented visual stimuli. The use of sequential instead of simultaneously presented stimuli is unlikely to explain the different dependence of contextual modulation on stimulus intensity in visual and auditory cortex. In visual and somatosensory cortex, sequentially presented stimuli can evoke facilitation and suppression similar to simultaneously presented stimuli (Lorenceau et al. 2001; Simons 1985). Sequential stimuli can also evoke facilitation and suppression of contrast thresholds in visual psychophysical studies (Tadin et al. 2006; Watson and Nachmias 1977). Typically, however, studies of contextual modulation in vision do not use sequential stimuli to avoid apparent motion. Likewise studies of contextual modulation in auditory cortex typically do not use simultaneous stimuli to avoid amplitude and phase modulations that can strongly affect neuronal responses (Schulze and Langner 1999). Reducing the interval between masker and probe would be unlikely to reveal facili- 
tation because forward suppression is maximal for the shortest masker-probe intervals (as short as $1 \mathrm{~ms}$ ) and decreases as this interval gets longer (Fitzpatrick et al. 1999; Wehr and Zador 2005). Nearly simultaneous stimuli are therefore likely to evoke even more suppression than we observed rather than evoke facilitation. In somatosensory cortex, facilitation can be seen within a narrow range of very short intervals $(5 \mathrm{~ms}$ on average) (Shimegi et al. 1999), intervals that in auditory cortex invariably lead to profound suppression (Fitzpatrick et al. 1999). This may reflect a fundamental difference in processing strategies between the somatosensory system and the auditory system. In somatosensation, fast facilitation could optimize texture discrimination in low signal-to-noise conditions, as neighboring vibrissae sequentially encounter a surface during a whisk. In audition, by contrast, the presence of surface reflections in natural acoustic environments greatly complicates the task of sound localization. Perceptual echo suppression, in which only the first sound is perceived for intervals shorter than the "echo threshold," demonstrates that the auditory system successfully solves this problem. Strong forward suppression of neuronal responses, especially for intervals $<10$ ms, may serve as a mechanism for echo suppression to ensure accurate sound localization.

Suppression serves a wide variety of functional roles in different sensory systems, but one computational requirement common to all modalities is the need for gain control. In the visual system, suppression has been interpreted as gain control acting to reduce either the input to a neuron or the output from that neuron (or pool of neurons) (Sengpiel et al. 1998). Suppression acting on inputs would shift the response-level function to the right, acting to reduce the effective stimulus level (curve L in Fig. 10A). In the visual system, this is referred to as subtractive or contrast-gain control; here we refer to its auditory analog as level-gain control. Suppression acting instead on neuronal output would scale the response-level function, acting to reduce response magnitude (curve $\mathrm{R}$ in Fig. $10 A$ ). This is referred to as divisive or response-gain control. Facilitation presumably acts to increase response magnitudes (curve $\mathrm{F}$ in Fig. 10A). The cross-over from facilitation of low-contrast central stimuli to suppression for high-contrast central stimuli (Moore et al. 1999; Polat et al. 1998; Toth et al. 1996) is depicted in Fig. $10 B$ as a combination of facilitation and response-gain control (i.e., curves $\mathrm{F}$ and $\mathrm{R}$, although the mechanisms underlying the cross-over are not fully understood). Our results for auditory cortical neurons are depicted in Fig. $10 C$ as a combination of response-gain control and levelgain control (i.e., curves $\mathrm{R}$ and L, compare with Figs. $4 A$ and $8 A$ ). The fact that we observed maximal suppression for intermediate probe levels suggests a major contribution from level-gain control. We suspect that response-gain control also contributes to forward suppression because even the highest probe levels remained suppressed, suggesting a lower asymptotic response (e.g., curve R). However, to clearly distinguish between the profiles of response-gain control and level-gain control would require additional data for probe levels substantially higher than those we used; such sound levels (which would be well above $100 \mathrm{~dB}$ ) are probably outside of the physiological range. We conclude that forward suppression probably includes contributions from both response-gain control and level-gain control. In contrast, forward suppression of responses to different binaural level combinations appears to act by response-gain control and not level-gain control (Nakamoto et al. 2006; Zhang et al. 2005). Although our free-field stimulus reached both ears, we did not independently vary binaural levels and thus do not know whether the binaural response properties of our neurons showed response-gain control, level-gain control, or both. Interestingly, both of these functional types of gain control can be explained by synaptic depression at thalamocortical synapses (Carandini et al. 2002).

The perceptual implications of these results are consistent with perceptual forward masking, in which maskers increase the threshold for probe detection (Moore 2003). Likewise the forward suppression we observed is consistent with the perceptual loudness decrement evoked by maskers for relatively high-level probes (Elmasian et al. 1980; Oberfeld 2007). However, we observed even greater suppression in auditory cortical neurons for relatively low-level probes; this is inconsistent with the perceptual loudness enhancement seen for relatively low-level probes (Galambos et al. 1972; Oberfeld 2007). There are at least three possible explanations for this discrepancy. First, loudness enhancement may have neural correlates in areas of the auditory system other than primary auditory cortex. Although we and others observed facilitation in A1 neurons (Brosch and Schreiner 2000; Brosch et al. 1999), this facilitation does not show the dependence on probe level seen with perceptual loudness enhancement and is therefore unlikely to underlie it. Second, increased perceptual sensitivity may depend on orienting or attentional effects not present in the anesthetized preparation. Finally, it has been argued that loudness enhancement may be an artifact due to loudness recalibration (Arieh and Marks 2003). Because loudness enhancement is measured by matching the probe loudness to the loudness of a comparison tone, apparent loudness enhancement could arise from a loudness decrement of the comparison tone. Our findings that contextual interactions are predominantly suppressive in auditory cortex suggest that this may also be true for their effects in perception.
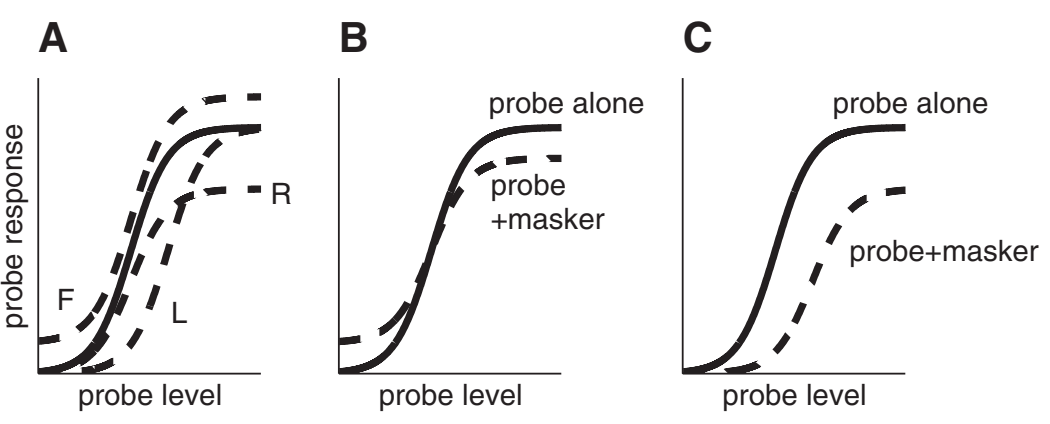

FIG. 10. Forward suppression and gain control mechanisms. A: - , neural response as a function of probe level, e.g., an auditory rate-level function or a visual contrast-response function. Level-gain control (curve L) results from subtractive suppression that shifts the response curve to the right. Response-gain control (curve R) results from divisive suppression that scales the response curve. Facilitation (curve F) could act by shifting the response curve upwards. $B$ : cross-over from facilitation for low probe levels to suppression for high probe levels as seen in visual and somatosensory cortex, modeled as a combination of curves $\mathrm{F}$ and $\mathrm{R}$. $C$ : a combination of responsegain control and level-gain control (curves $\mathrm{R}$ and L), consistent with the suppression we observed in auditory cortical cells.

J Neurophysiol • VOL 99 • APRIL 2008 • www.jn.org 


\section{REFERENCES}

Albright TD, Stoner GR. Contextual influences on visual processing. Annu Rev Neurosci 25: 339-379, 2002.

Allman J, Miezin F, McGuinness E. Stimulus specific responses from beyond the classical receptive field: neurophysiological mechanisms for local-global comparisons in visual neurons. Annu Rev Neurosci 8: 407-430, 1985.

Angelucci A, Levitt JB, Walton EJ, Hupe JM, Bullier J, Lund JS. Circuits for local and global signal integration in primary visual cortex. $J$ Neurosci 22: 8633-8646, 2002.

Arieh Y, Marks LE. Time course of loudness recalibration: implications for loudness enhancement. J Acoust Soc Am 114: 1550-1556, 2003.

Azouz R, Gray CM. Cellular mechanisms contributing to response variability of cortical neurons in vivo. J Neurosci 19: 2209-2223, 1999.

Bartlett EL, Wang X. Long-lasting modulation by stimulus context in primate auditory cortex. J Neurophysiol 94: 83-104, 2005.

Bringuier V, Chavane F, Glaeser L, Fregnac Y. Horizontal propagation of visual activity in the synaptic integration field of area 17 neurons. Science 283: 695-699, 1999.

Brosch M, Schreiner CE. Time course of forward masking tuning curves in cat primary auditory cortex. J Neurophysiol 77: 923-943, 1997.

Brosch M, Schreiner CE. Sequence sensitivity of neurons in cat primary auditory cortex. Cereb Cortex 10: 1155-1167, 2000.

Brosch M, Schulz A, Scheich H. Processing of sound sequences in macaque auditory cortex: response enhancement. J Neurophysiol 82: 1542-1559, 1999.

Brumberg JC, Pinto DJ, Simons DJ. Spatial gradients and inhibitory summation in the rat whisker barrel system. J Neurophysiol 76: 130$140,1996$.

Calford MB, Semple MN. Monaural inhibition in cat auditory cortex. $J$ Neurophysiol 73: 1876-1891, 1995.

Carandini M, Ferster D. Membrane potential and firing rate in cat primary visual cortex. J Neurosci 20: 470-484, 2000.

Carandini M, Heeger DJ, Senn W. A synaptic explanation of suppression in visual cortex. J Neurosci 22: 10053-10065, 2002.

Castro-Alamancos MA. Role of thalamocortical sensory suppression during arousal: focusing sensory inputs in neocortex. J Neurosci 22: 9651-9655, 2002.

DeWeese MR, Wehr M, Zador AM. Binary spiking in auditory cortex. J Neurosci 23: 7940-7949, 2003.

Elmasian R, Galambos R, Bernheim A, Jr. Loudness enhancement and decrement in four paradigms. J Acoust Soc Am 67: 601-607, 1980.

Fitzpatrick DC, Kuwada S, Kim DO, Parham K, Batra R. Responses of neurons to click-pairs as simulated echoes: auditory nerve to auditory cortex. J Acoust Soc Am 106: 3460-3472, 1999.

Galambos R, Bauer J, Picton T, Squires K, Squires N. Loudness enhancement following contralateral stimulation. J Acoust Soc Am 52: 1127-1130, 1972.

Ghazanfar AA, Nicolelis MA. Nonlinear processing of tactile information in the thalamocortical loop. J Neurophysiol 78: 506-510, 1997.

Harris DM, Dallos P. Forward masking of auditory nerve fiber responses. J Neurophysiol 42: 1083-1107, 1979.

Higley MJ, Contreras D. Integration of synaptic responses to neighboring whiskers in rat barrel cortex in vivo. J Neurophysiol 93: 1920-1934, 2005.

Higley MJ, Contreras D. Cellular mechanisms of suppressive interactions between somatosensory responses in vivo. J Neurophysiol 97: 647-658, 2007.

Hirsch JA, Gilbert CD. Synaptic physiology of horizontal connections in the cat's visual cortex. J Neurosci 11: 1800-1809, 1991.

Horikawa J, Hosokawa Y, Nasu M, Taniguchi I. Optical study of spatiotemporal inhibition evoked by two-tone sequences in the guinea pig auditory cortex. J Comp Physiol [A] 181: 677-684, 1997.

Kaur S, Lazar R, Metherate R. Intracortical pathways determine breadth of subthreshold frequency receptive fields in primary auditory cortex. $J \mathrm{Neu}$ rophysiol 91: 2551-2567, 2004.

Levitt JB, Lund JS. Contrast dependence of contextual effects in primate visual cortex. Nature 387: 73-76, 1997.

Lorenceau, Baudot, Series, Georges, Pananceau, Fregnac. Modulation of apparent motion speed by horizontal intracortical dynamics. J Vision 1: 400, 2001.
Malone BJ, Semple MN. Effects of auditory stimulus context on the representation of frequency in the gerbil inferior colliculus. J Neurophysiol 86: 1113-1130, 2001.

Micheyl C, Carlyon RP, Gutschalk A, Melcher JR, Oxenham AJ, Rauschecker JP, Tian B, Courtenay Wilson E. The role of auditory cortex in the formation of auditory streams. Hear Res 229: 116-131, 2007.

Moore BCJ. An Introduction to the Psychology of Hearing (5th ed.). San Diego, CA: Academic, 2003.

Moore CI, Nelson SB. Spatio-temporal subthreshold receptive fields in the vibrissa representation of rat primary somatosensory cortex. J Neurophysiol 80: 2882-2892, 1998.

Moore CI, Nelson SB, Sur M. Dynamics of neuronal processing in rat somatosensory cortex. Trends Neurosci 22: 513-520, 1999.

Nakamoto KT, Zhang J, Kitzes LM. Temporal nonlinearity during recovery from sequential inhibition by neurons in the cat primary auditory cortex. J Neurophysiol 95: 1897-1907, 2006.

Nelson JI, Frost BJ. Orientation-selective inhibition from beyond the classic visual receptive field. Brain Res 139: 359-365, 1978.

Oberfeld D. Loudness changes induced by a proximal sound: loudness enhancement, loudness recalibration, or both? J Acoust Soc Am 121: 2137 2148, 2007.

Ojima H, Murakami K. Intracellular characterization of suppressive responses in supragranular pyramidal neurons of cat primary auditory cortex in vivo. Cereb Cortex 12: 1079-1091, 2002.

Patuzzi R, Sellick PM, Johnstone BM. The modulation of the sensitivity of the mammalian cochlea by low frequency tones. III. Basilar membrane motion. Hear Res 13: 19-27, 1984.

Phillips DP, Cynader MS. Some neural mechanisms in the cat's auditory cortex underlying sensitivity to combined tone and wide-spectrum noise stimuli. Hear Res 18: 87-102, 1985.

Polat U, Mizobe K, Pettet MW, Kasamatsu T, Norcia AM. Collinear stimuli regulate visual responses depending on cell's contrast threshold. Nature 391: 580-584, 1998.

Polat U, Sagi D. Temporal asymmetry of collinear lateral interactions. Vision Res 46: 953-960, 2006.

Reale RA, Brugge JF. Directional sensitivity of neurons in the primary auditory (AI) cortex of the cat to successive sounds ordered in time and space. J Neurophysiol 84: 435-450, 2000.

Schulze H, Langner G. Auditory cortical responses to amplitude modulations with spectra above frequency receptive fields: evidence for wide spectral integration. J Comp Physiol [A] 185: 493-508, 1999.

Sengpiel F, Baddeley RJ, Freeman TC, Harrad R, Blakemore C. Different mechanisms underlie three inhibitory phenomena in cat area 17. Vision Res 38: 2067-2080, 1998.

Sengpiel F, Sen A, Blakemore C. Characteristics of surround inhibition in cat area 17. Exp Brain Res 116: 216-228, 1997.

Shamma SA, Symmes D. Patterns of inhibition in auditory cortical cells in awake squirrel monkeys. Hear Res 19: 1-13, 1985.

Shao Z, Burkhalter A. Different balance of excitation and inhibition in forward and feedback circuits of rat visual cortex. J Neurosci 16: 7353$7365,1996$.

Shimegi S, Ichikawa T, Akasaki T, Sato H. Temporal characteristics of response integration evoked by multiple whisker stimulations in the barrel cortex of rats. J Neurosci 19: 10164-10175, 1999.

Simons DJ. Temporal and spatial integration in the rat SI vibrissa cortex. J Neurophysiol 54: 615-635, 1985.

Somers DC, Todorov EV, Siapas AG, Toth LJ, Kim DS, Sur M. A local circuit approach to understanding integration of long-range inputs in primary visual cortex. Cereb Cortex 8: 204-217, 1998.

Sutter ML, Schreiner CE. Physiology and topography of neurons with multipeaked tuning curves in cat primary auditory cortex. $J$ Neurophysiol 65: 1207-1226, 1991.

Sutter ML, Schreiner CE, McLean M, O'Connor KN, Loftus WC. Organization of inhibitory frequency receptive fields in cat primary auditory cortex. J Neurophysiol 82: 2358-2371, 1999.

Tadin D, Lappin JS, Blake R. Fine temporal properties of center-surround interactions in motion revealed by reverse correlation. J Neurosci 26: 2614-2622, 2006.

Tai L-H, Zador AM. In vivo whole cell recording of synaptic responses underlying two-tone interactions in rat auditory cortex. Soc Neurosci Abstr 27: 621.1, 2001. 
Tan AY, Zhang LI, Merzenich MM, Schreiner CE. Tone-evoked excitatory and inhibitory synaptic conductances of primary auditory cortex neurons. J Neurophysiol 92: 630-643, 2004.

Toth LJ, Rao SC, Kim DS, Somers D, Sur M. Subthreshold facilitation and suppression in primary visual cortex revealed by intrinsic signal imaging. Proc Natl Acad Sci USA 93: 9869-9874, 1996.

Watson AB, Nachmias J. Patterns of temporal interaction in the detection of gratings. Vision Res 17: 893-902, 1977.
Wehr M, Zador AM. Balanced inhibition underlies tuning and sharpens spike timing in auditory cortex. Nature 426: 442-446, 2003

Wehr M, Zador AM. Synaptic mechanisms of forward suppression in rat auditory cortex. Neuron 47: 437-445, 2005.

Zhang J, Nakamoto KT, Kitzes LM. Modulation of level response areas and stimulus selectivity of neurons in cat primary auditory cortex. J Neurophysiol 94: 2263-2274, 2005. 